\title{
A Case report of a Knee Tuberculosis- arthritis in a mid aged lady in India
}

\section{Srishti Basu}

Jilin University

Dattatreya Mukherjee ( $\nabla$ dattatreyamukherjee4u@outlook.com )

Jinan University https://orcid.org/0000-0001-7566-3843

\section{Case Report}

Keywords: knee, tuberculosis, extrapulmonary tuberculosis

Posted Date: October 12th, 2020

DOI: https://doi.org/10.21203/rs.3.rs-90729/v1

License: (c) (i) This work is licensed under a Creative Commons Attribution 4.0 International License. Read Full License 


\section{A Case report of a Knee Tuberculosis- arthritis in a mid aged lady in India}

Srishti Basu ${ }^{(1)}$, Dattatreya Mukherjee ${ }^{(2)}$

1. Jilin University, P.R China

2. Jinan University, P.R China

Corresponding author: Dattatreya Mukherjee

dattatreyamukherjee4u@outlook.com

Abstract:

Tuberculosis (TB) is a common disease in developing countries but now days the incidence decreased due to the community use of BCG vaccine after birth. A 55 years old female contacted through tele-medicine with history of Right knee pain for last 3 months. CT and MRI of Right knee is referred. The CT and MRI suggested the Knee Tuberculosis(Rice body Appearance) so the patient is referred to the near by internal Orthopedic department for anti tuberculosis treatment.

\section{Introduction:}

Tuberculosis is a very common disease and endemic disease in Developing Countries(1). India has the $24 \%$ of global prevalence, $23 \%$ of global incidence and $21 \%$ of total TB death.(2) The most common tuberculosis is pulmonary TB. Exptra pulmonary TB (EPTB) is not common now days. Bone TB accounts the $11.2 \%$ of total EPTB. In bone, knee, spine and hip affect mostly by micro bacterium tuberculosis. Rice Body lesion can be present in RA, TB arthritis and fungal diseases, gout, SLE etc. $(3,4)$

Here we presented a case of a right Knee Tuberculosis - arthritis.

Case:

A 55 year old woman presents to the local orthopedic clinic with right knee pain since the last 2 months .CT and MRI are advised to the patient, on which, a rice body appearance is seen. The patient is HIV seronegative. There is no history of any chronic diseases or recent trauma. The patient has not undergone any analgesic medication course. Any particular gait disturbances are absent.

CT scan reveals minimal knee joint effusion with a small pocket of air in medial compartment of tibio-femoral joint. The articular margins are smooth. The femur, tibia, fibula and patella appear normal. Any evidence of any fractures or focal lesions is missing. The muscles appear normal too on CT, with normal intermuscular planes. The presence of any soft tissue component or fluid collection is also lacking. But there is mild subcutaneous edema seen in the lateral aspect of right knee.

Magnetic resonance imaging reveals enhancing, T1 hypo intense signals and STIR (Short tau inversion recovery) hyper intense marrow lesions. 
The MRI also reveals cortical destruction at medial and lateral femoral condyles and medical tibial condyle of the right knee .However, the destruction at medial femoral condyles is more prominent than the lateral one. Furthermore, adjoining soft tissue inflammation and associated right knee joint effusion with intense, irregularly enhancing synovial thickening of right knee is observed on MRI. In addition, edema, thinning and laxity of anterior cruciate ligament of right knee is observed. The appearances were suggestive of chronic tubercular arthritis or synovitis of right knee.

The patient was tested AFB positive for sputum sample. A synovial biopsy was advised and anti-tubercular drug regimen was started according to the RNTCP (Revised National TB Control Programme). A six month treatment regimen is advised to her which comprises of a 2 month long Intensive phase and 4 month long continuous phase. While in the intensive phase of her treatment, she is on HRZE drugs i.e. Isoniazid, Rifampicin, Pyrazinamide and Ethambutol respectively. The continuous phase consists of HRE medications i.e. Isoniazid, Rifampicin and Ethambutol.

The patient is being reviewed while on the anti-TB treatment regimen. Currently the patient is under consideration for knee replacement surgery. 


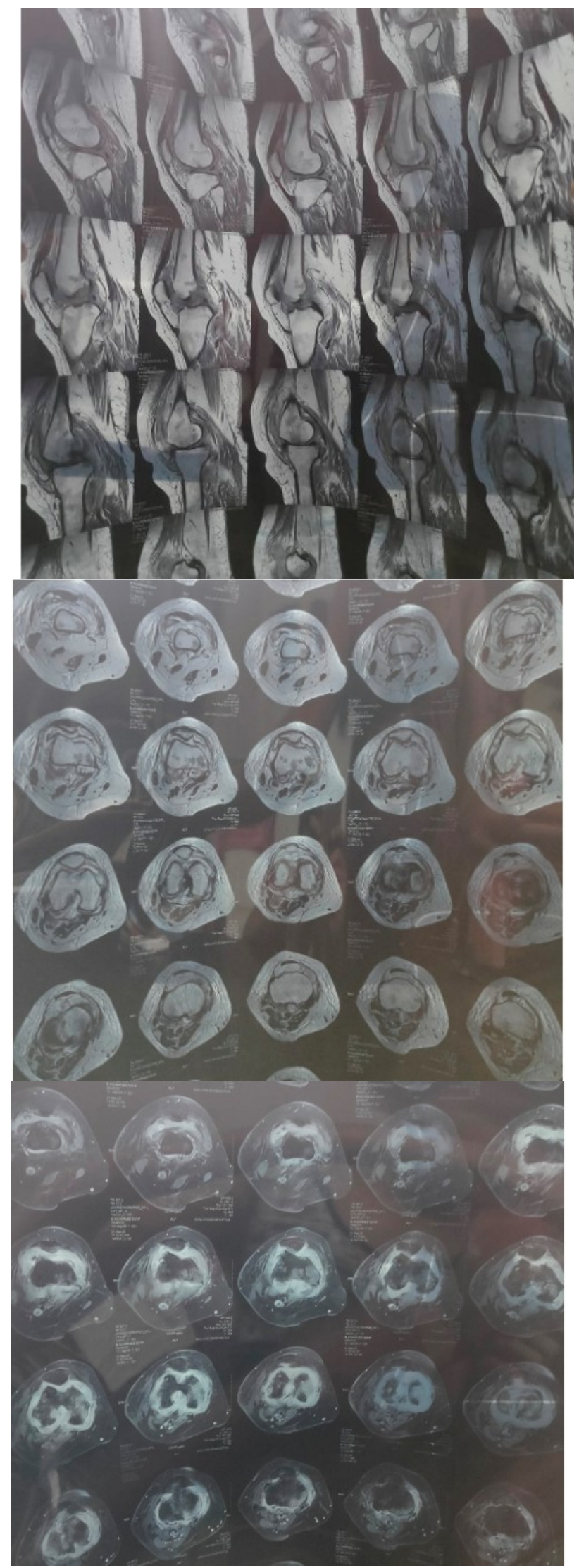

MRI and CT of the Right Knee suggesting knee TB arthritis 


\section{Discussion:}

TB is extremely common in developing countries like India. According to the World Health Organization (WHO) TB Statistics for India for 2018, the estimated incidence figure is of 2.69 million cases. The TB incidence is the number of new cases of active TB disease during a certain time period, usually a year. The mortality of TB patients (excludes HIV+TB) is $4,40,000(0.72 \text { per } 100,000 \text { population })^{5}$.About $40 \%$ of the Indian population is infected with TB bacteria, the vast majority of whom have latent TB rather than TB disease. The usual clinical presentation of knee TB is gradual pain, limp, flexion and synovitis. Our 55 year old female patient too presented with a 2 month history of knee pain and limp .A 'triple deformity' consisting of posterior subluxation of tibia, external rotation of leg and flexion of knee is also seen in such cases. However, the triple deformities may also be a feature of rheumatoid arthritis, iliotibial band contracture, polio and others.

TB is caused by Mycobacterium tuberculosis which is $20 \%$ acid fast (due to mycolic acid). Mycobacterium tuberculosis usually has 3 main virulence factors, namely cord factor, lipoarabinomannan and HSP (Heat shock protein). For the definitive diagnosis of TB knee or tubercular arthritis, Mycobacterium tuberculosis has to be isolated and cultured ${ }^{6}$. In this case, too, after the initial MRI and CT scan reports were indicative of knee TB, a sputum sample was taken and the patient tested AFB positive. The management of TB knee consists of a combination therapy drug regimen that continues for few months and is divided into the intensive phase (IP) and continuous phase (CP). ${ }^{7}$ In a patient like ours, who has not undergone any previous treatment or is not a treatment failure, defaulter or a relapsed case, we administer HRZE for 2 months (IP) and HRE for 4 months (CP) .The total duration of treatment would therefore be of 6 months. In patients who are previous treatment failures, defaulters or have had relapses, the treatment duration is of 8 months which is divided into 2 months of HRZES(S-Streptomycin), 1 month of HRZE in the IP followed by 5 months of HRE in the CP. The treatment regimen for MDR TB is even longer, going up to $18-27$ months .The IP consists of KLCZEEt (K-Kanamycin, L-Leviflux, C-Cyclosporine,ZPyrizinamide,E-Ethambutol,Et-Ethionamide) for 6-9 months and the $\mathrm{CP}$ consists of LCEEt(L-Linezolid-Capreomycin) for 12-18 months.

Surgical options are also commonly opted for in cases of TB knee like arthrotomy followed by curettage of the lesion. ${ }^{7}$

\section{Conclusion:}

Its a case of Knee TB arthritis. Treatment is still going on. Doctors are also thinking to do a knee replacement surgery if the prognosis is not good. 
Funding: No funding is related to this research

Patient/ Family member Consent: Taken

Conflict of Interest: No Conflict of Interest

\section{References:}

1. J. Amini, H. Poka, J. Kumbu et al., "The crisis of tuberculosis in Papua New Guinea-the role of older strategies for public health disease control," Papua and New Guinea Medical Journal, vol. 55, no. 1-4, pp. $1-4,2012$

2. World Health Organization. Annual TB Report. World Health Organization; 2015. Tuberculosis Control in the South East Asia Region 3. M. Tyllianakis, G. Kasimatis, S. Athanaselis, and M. Melachrinou, "Rice-body formation and tenosynovitis of the wrist: a case report," Journal of Orthopaedic Surgery, vol. 14, no. 2, pp. 208-211, 2006.

4. S. Bayram, A. Erşen, M. Altan, and H. Durmaz, "Tuberculosis tenosynovitis with multiple rice bodies of the flexor tendons in the wrist: a case report," International Journal of Surgery Case Reports, vol. 27, pp. 129-132, 2016

5. World Health Organization TB Statistics for India . (accessed 8/10/2020); https://tbfacts.org/tb-statistics-india/

6.Lidder, Surjit et al. "Tuberculosis of the knee." Orthopedic reviews vol. 1,2 (2009): e24. doi:10.4081/or.2009.e24

7. Uboldi, Francesco M et al. "Tuberculosis of the Knee: A Case Report and Literature Review." Joints vol. 5,3 180-183. 11 Aug. 2017, doi:10.1055/s-0037-1605591 


\section{Figures}

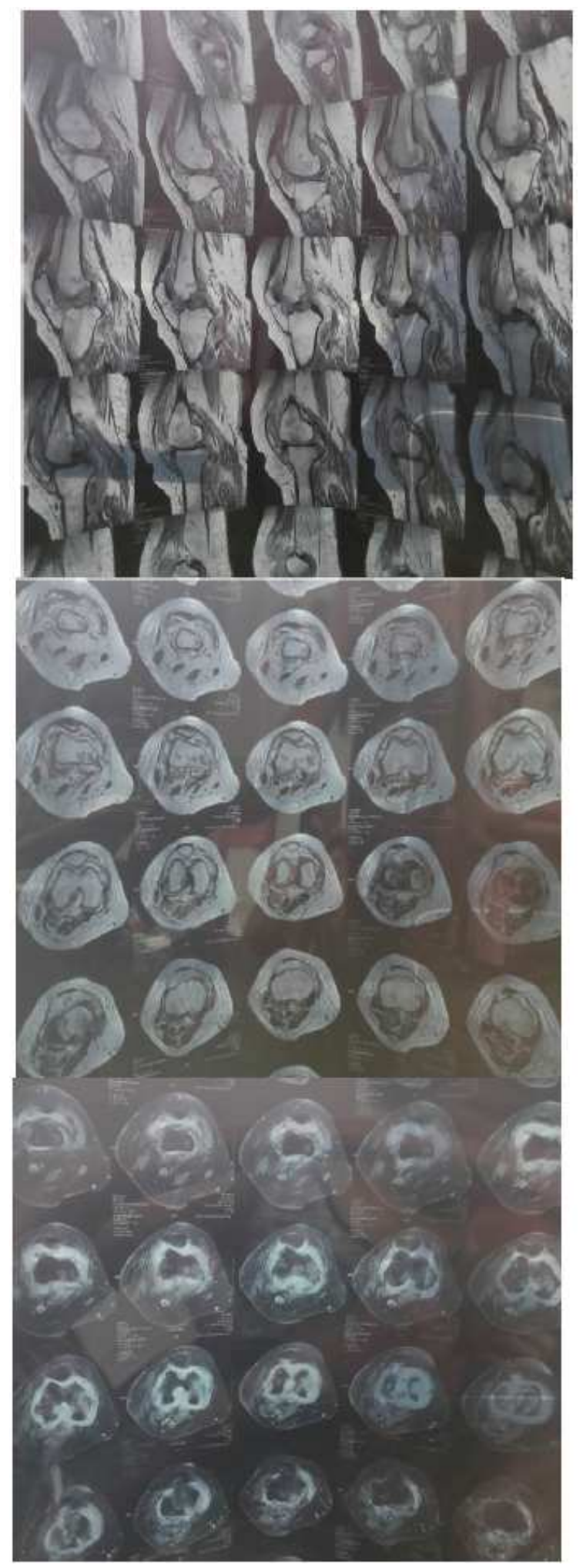

Figure 1

MRI and CT of the Right Knee suggesting knee TB arthritis 